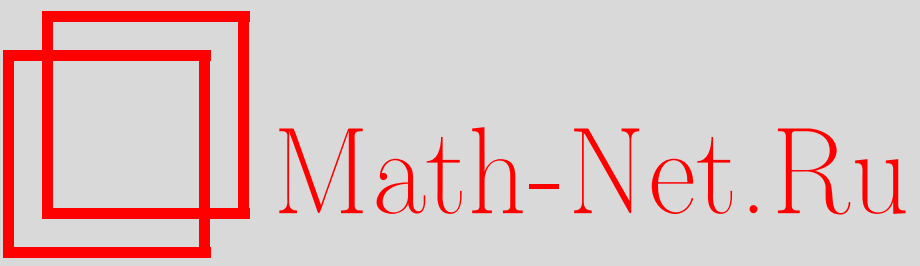

А. В. Болсинов, Инварианты Фоменко в теории интегрируемых гамильтоновых систем, УМН, 1997, том 52, выпуск 5, 113-132

DOI: https://doi.org/10.4213/rm886

Использование Общероссийского математического портала Math-Net.Ru подразумевает, что вы прочитали и согласны с пользовательским соглашением

http://www . mathnet.ru/rus/agreement

Параметры загрузки:

IP : 3.89 .185 .249

26 апреля 2023 г., 18:23:49

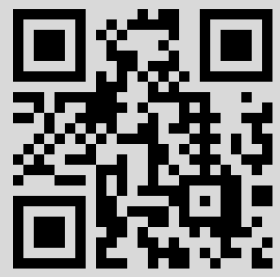




\section{ИНВАРИАНТЫ ФОМЕНКО В ТЕОРИИ ИНТЕГРИРУЕМЫХ ГАМИЛЬТОНОВЫХ СИСТЕМ}

А. В. Болсинов

СОДЕРЖАНИЕ

Введение

Случай одной степени свободы, функции Морса на двумерных поверхностях, атомы, градиентоподобные потоки ................... 116

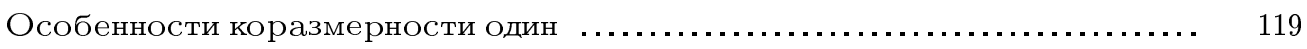

Случай двух степеней свободы, изоэнергетические поверхности, моле-

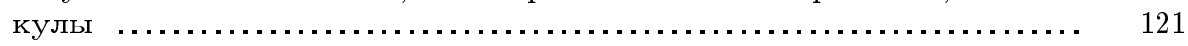

Круговые молекулы и гипотеза Фоменко . . . . . . . . . . . . . . . . . . . . . 123

Невырожденные особенности в случае многих степеней свободы. Теорема о топологическом разложении ........................ 124

Траекторная классификация интегрируемых гамильтоновых систем $\ldots . .127$

Задача Якоби о геодезических на эллипсоиде и случай Эйлера в динамике твердого тела ......................................... 128

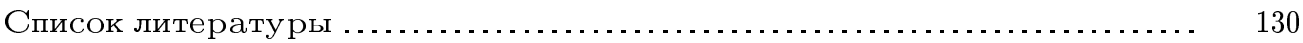

\section{Введение}

В этой работе мы хотели бы рассказать о некоторых недавних результатах в топологии интегрируемых гамильтоновых систем, основанных на применении нового подхода, предложенного А. Т. Фоменко [1], [2] около 10 лет назад.

Этот подход возник как аналог теории Морса для интегрируемых гамильтоновых систем и имел целью описать типичные бифуркации торов Лиувилля, топологию изоэнергетических поверхностей таких систем и получить на этой основе новые топологические препятствия к интегрируемости. В дальнейшем развитие этих идей оказалось весьма полезным для топологического анализа классических интегрируемых случаев в геометрии, механике и физике, описания структуры особенностей многомерных лиувиллевых слоений и их глобальных инвариантов. Были найдены интересные приложения к трехмерной топологии и симплектической геометрии. Наконец, в последние годы в рамках этого обшего подхода были обнаружены новые траекторные инварианты интегрируемых гамильтоновых систем с двумя степенями свободы, позволившие 
получить их классификацию с точностью до гомео- и диффеоморфизмов, сохраняющих траектории.

Рассмотрим четномерное симплектическое многообразие $\left(M^{2 n}, \omega\right)$ и гамильтонову систему на нем, порожденную гладким гамильтонианом $H: M \rightarrow \mathbb{R}$,

$$
\frac{d x}{d t}=X_{H}(x)
$$

Здесь $X_{H}=\operatorname{sgrad} H-$ векторное поле, однозначно определяемое соотношением $\omega\left(X_{H}, v\right)=v(H)$, где $v-$ произвольное векторное поле.

ОПРЕДЕЛЕНИЕ 1. Гамильтонова система $\left(X_{H}, M^{2 n}\right)$ назьвается вполне интегрируемой по Лиувиллю, если она допускает $n$ попарно коммутирующих первых интегралов $f_{1}, f_{2}, \ldots, f_{n}$, которые функционально независимы на $M^{2 n}$ почти всюду.

Напомним знаменитую теорему Лиувилля, описьвающую качественное поведение интегральных траекторий интегрируемых гамильтоновых систем.

ТЕОРЕМА ЛИУВИЛЛЯ. Если совместная поверхность уровня первых интегралов $L=\left\{f_{1}=c_{1}, f_{2}=c_{2}, \ldots, f_{n}=c_{n}\right\}$ является неособой, компактной $u$ связной, то L - это п-мерный тор $T^{n}$ (называемьй тором Лиувилля), причем в некоторой его окрестности $U(L)=T^{n} \times D^{n}$ существует каноническая система координат действие-угол $\left(s_{1}, \ldots, s_{n}, \varphi_{1}, \ldots, \varphi_{n}\right)$ такая, что

1) $s_{1}, \ldots, s_{n}-$ координатын на диске $D^{n}$ и $s_{i}=s_{i}\left(f_{1}, \ldots, f_{n}\right)$;

2) $\varphi_{1}, \ldots, \varphi_{n}-$ стандартные угловые координаты на торе;

3) $X_{H}=\sum a_{i} \frac{\partial}{\partial \varphi_{i}}$, əде $a_{i}=a_{i}\left(s_{1}, \ldots, s_{n}\right)$.

Таким образом, в случае интегрируемости на симплектическом многообразии возникает структура так назьваемого слоения Лиувилля $\mathscr{L}$, слоями которого по определению являются связные компоненты совместных поверхностей уровня первых интегралов $f_{1}, \ldots, f_{n}$. Каждое интегральное многообразие $L=\left\{f_{1}=c_{1}, f_{2}=c_{2}, \ldots, f_{n}=c_{n}\right\}$ (как особое, так и не особое) инвариантно относительно гамильтонова потока и расслоено на его траектории. Поэтому для понимания структуры фазового портрета в целом нам, грубо говоря, нужно решить две задачи: описать структуру лиувиллева слоения, а затем описать поведение траекторий на каждом его слое.

Ниже мы будем всегда предполагать, что отображение момента $\mathscr{F}=\left(f_{1}, \ldots, f_{n}\right)$ : $M^{2 n} \rightarrow \mathbb{R}^{n}$ является собственным, т.е. прообраз каждого компактного подмножества является компактным. В частности, все слои лиувиллева слоения компактны, и почти все они в силу теоремы Лиувилля являются $n$-мерньми торами. Однако, существуют и особые слои, отвечаюшие критическим значениям отображения момента $\mathscr{F}$.

Одной из основных задач топологии интегрируемых гамильтоновых систем является описание структуры лиувиллевых слоений, их особенностей и инвариантов, а также классификация таких слоений с точностью до естественного отношения эквивалентности.

ОПРЕДЕЛЕНИЕ 2. Два лиувиллевых слоения $\mathscr{L}_{1}$ и $\mathscr{L}_{2}$, отвечающие интегрируемым гамильтоновым системам $\left(X_{H_{1}}, M_{1}\right)$ и $\left(X_{H_{2}}, M_{2}\right)$ соответственно, называются әквивалентными, если сушествует послойньй гомеоморфизм (или дифффеоморфизм) 
$\xi: M_{1} \rightarrow M_{2}$. В этой случае мы, кроме того, говорим, что сами системы $\left(X_{H_{1}}, M_{1}\right)$ и $\left(X_{H_{2}}, M_{2}\right)$ лиувиллево әквивалентнь.

Вместо симплектических многообразий $M_{1}$ и $M_{2}$ в этом определении можно рассматривать любые их части, инвариантные относительно гамильтонова потока, например, изоэнергетические поверхности $Q_{i}=\left\{H_{i}=\right.$ const $\}$ или насышенные окрестности особых слоев.

Здесь следует сделать еще одно важное замечание. Определение лиувиллева слоения зависит, вообше говоря, от выбора дополнительных первых интегралов. Однако, если рассматриваемая гамильтонова система нерезонансна, т.е. на почти всех торах Лиувилля траектории являются всюду плотными обмотками, то почти все слои могут быть охарактеризованы в терминах самого гамильтонова потока $X_{H}$ как замыкания таких траекторий. Поэтому в типичной ситуации структура лиувиллева слоения не зависит от выбора дополнительных интегралов.

С другой стороны, можно было бы несколько видоизменить определение, чтобы сразу избавиться от этой зависимости. Скажем, что две точки $x, y \in M^{4}$ эквивалентны, если $f(x)=f(y)$ для любого гладкого первого интеграла $f$. Тогда лиувиллевым слоением можно было бы называть разбиение многообразия на классы такой эквивалентности. Это определение на самом деле не равносильно предыдушему. Например, в случае резонансной системы слои общего положения могут иметь размерность меньшую $n=1 / 2 \operatorname{dim} M$. Для простоты нам будет удобно тем не менее пользоваться исходньм определением, т.е. предполагать, что первые интегралы гамильтоновой системы фиксированы.

Отметим, что задачу об описании структуры лиувиллева слоения можно было бы переформулировать следуюшим образом. Коммутирующие первые интегралы $f_{1}, \ldots, f_{n}$ задают на симплектическом многообразии гамильтоново действие группы $\mathbb{R}^{n}$. Требуется классифищировать такие действия с точностью до орбитальной эквивалентности. Напомним, что два действия назьваются орбитально эквивалентными, если сушествует гомеоморфизм (или диффеоморфизм), переводящий орбиты в орбиты.

Отметим, что структура лиувиллевых слоений была исследована в двух важных частных случаях, а именно, был исследован случай лиувиллева слоения без особенностей (J. J. Duistermaat [27]) и случай пуассонова действия тора (см. книгу М. Au$\operatorname{din}[28])$.

Кроме описания структуры лиувиллевых слоений имеется еще одна (и может быть, даже более интересная) проблема, состояшая в нахождении топологических препятствий к интегрируемости. Другими словами, наиболее обший вопрос может быть сформулирован следуюшим образом: какие симплектические многообразия допускают интегрируемые по Лиувиллю интегрируемые гамильтоновы системы?

ЗАмечАниЕ. На самом деле этот вопрос нуждается в некотором уточнении. Дело в том, что можно построить пример интегрируемой гамильтоновой системы, гамильтониан и интегралы которой имеют в качестве носителя $2 n$-мерный шар, а вне этого шара тождественно равны нулю (см. [3]). Покрывая симплектическое многообразие $M^{2 n}$ непересекаюшимися шарами так, чтобы дополнение к ним имело меру нуль, и склеивая затем между собой гамильтонианы и интегралы из разных шаров, несложно построить гамильтонову систему, интегрируемую в целом на $M^{2 n}$. Однако, множес- 
тво особых точек отображения момента и, следовательно, множество особых слоев лиувиллева слоения будут иметь при этом слишком сложную структуру. В частности, ни гамильтониан, ни первые интегралы не будут функциями Морса. В этой конструкции они также не могут быть сделаны аналитическими. Поэтому имеет смысл ограничиться рассмотрением интегрируемых систем, обладающих некоторыми дополнительными свойствами. Например, можно требовать, чтобы гамильтониан был функцией Морса, или аналитической функцией, или предполагать, что особенности лиувиллева слоения устроены достаточно просто (например, все являются невырожденньми).

Начиная изучение сформулированных вьше общих вопросов, следует прежде всего описать структуру особенностей лиувиллевых слоений, в частности, типы бифуркаций торов Лиувилля и топологию особых слоев. Вопросы такого характера неоднократно возникали во многих работах, связанных как с чисто теоретическими конструкциями, так и с приложениями. Нам хотелось бы, в частности, упомянуть известную работу С. Смейла “Топология и механика" [4], а также книгу М. П. Харламова [5], в которой были исследованы свойства лиувиллевых слоений для интегрируемых случаев динамики твердого тела. В [5], кстати, после детального изучения большого числа примеров, имеющих на самом деле фундаментальный характер, автором была высказана надежда на скорое построение общей теории типа теории Морса, описьвающей структуру и особенности лиувиллевых слоений. Такая теория действительно была создана на основе нового подхода (и, можно сказать, нового языка), предложенного А.Т. Фоменко в 1985-86 гг. (см. [1]-[2], [6]-[8]). В этих работах, в частности, были решены две задачи:

1) получено описание невырожденных особенностей коразмерности один для лиувиллевых слоений произвольной размерности,

2) дана глобальная классификация лиувиллевых слоений на изоэнергетических поверхностях интегрируемых гамильтоновых систем с двумя степенями свободы.

Чтобы объяснить основные идеи и конструкции этого подхода, мы рассмотрим сначала очень простой пример.

\section{Случай одной степени свободы, функции Морса на двумерных поверхностях, атомы, градиентоподобные потоки}

Рассмотрим гамильтонову систему с одной степенью свободы на двумерной компактной поверхности $M^{2}$. Такая система, очевидно, всегда является интегрируемой, и структура лиувиллева слоения на $M^{2}$ задается в этом случае самим гамильтонианом $f: M^{2} \rightarrow \mathbb{R}$. Более точно, в этом случае лиувиллево слоение является слоением на связные компоненты линий уровня функции $f$. Невырожденность особенностей в этом случае означает, что $f$ - это функция Морса. Вместо классификации лиувиллевых слоений мы могли бы в этом случае говорить о топологической классификации функций Морса на двумерной поверхности, считая две функции топологически эквивалентными тогда и только тогда, когда существует диффеоморфизм, переводяший линии уровня одной функции в линии уровня другой.

Пусть $L$ - связная линия уровня функции $f$, содержашая одну или несколько особых точек. Ясно, что в общем случае особьй слой $L$ может быть либо изолированной 
точкой (являющейся локальным минимумом или локальным максимумом функции $f$ ), либо связным грабром, каждая вершина которого имеет степень 4 (простейшим примером является восьмерка).

Рассмотрим регулярную насышенную окрестность $P^{2}=U(L)$ особого слоя $L$. В качестве такой окрестности мы берем множество $f^{-1}(c-\varepsilon, c+\varepsilon)$, где $f(L)=c$, считая $\varepsilon$ достаточно малым для того, чтобы в эту окрестность не попало никаких других особых точек.

Ясно, что слой $L$ является деформационным ретрактом окрестности $P^{2}$. Вершины графа $L$ и только они являются особыми точками функции $\left.f\right|_{P^{2}}$. Множество $P^{2} \mathrm{~L}$ представляет собой набор колец, которые в седловом случае естественным образом разбиваются на два класса так, что к каждому ребру графа $L$ примькают кольца разных классов. Каждое из этих колец тривиальным образом расслоено на неособые слои, гомеоморфные окружности, поэтому структура слоения однозначно восстанавливается по паре $\left(P^{2}, L\right)$.

ОПРЕДЕЛЕНИЕ 3 . Окрестность $P^{2}$ особого слоя $L$ со структурой слоения Лиувилля на ней назьвается атомом. Сложностью атома называется число вершин графа $L$.

Другими словами, атомом называется тип особенности лиувиллева слоения в случае одной степени свободы. Мы подразумеваем здесь, что две особенности имеют одинаковый тип, если соответствуюшие слоения эквивалентны в некоторых окрестностях особых слоев.

Имеется несколько методов алгоритмической классификации атомов (см. [8], [9]). Они основаны на том, что число атомов фиксированной сложности конечно, и их полньй список в принципе может быть получен с помощью конечного алгоритма. На рис. 1 показаны все атомы сложности 1 и 2 и их условные обозначения, в [8] приведен более широкий список.

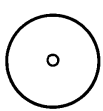

a) $A-$

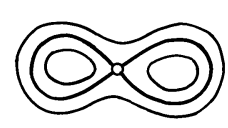

5) $-B=$

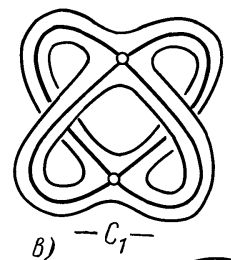

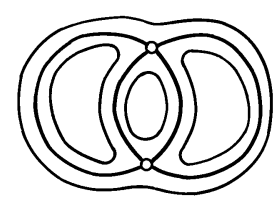

2) $>c_{2}=$ B) $-C_{1}-$
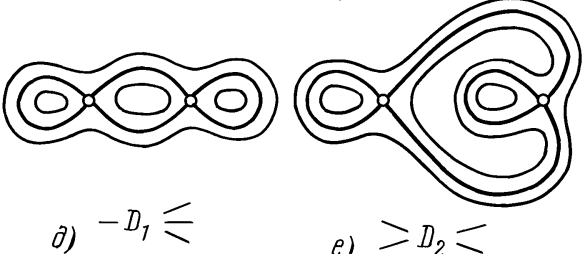

e) $>D_{2}=$

Рис. 1

Если мы хотим теперь описать структуру лиувиллева слоения в целом, то, после того как структура всех особых слоев (т.е. атомов) описана, сделать это несложно. Фактически достаточно указать, каким образом различные атомы примыкают друг 
к другу. Наглядно это можно сделать следуюшим образом. Рассмотрим сначалаграф Риба гамильтониана $f$, т.е. базу слоения Лиувилля. Вершины этого граф̆а соответствуют, очевидно, особым слоям. Каждой из них мы сопоставляем соответствующий атом. В результате получается объект, назьваемьй молекулой интегрируемой системь и даюший описание лиувиллева слоения в целом. Здесь есть некоторые тонкости, связанные с ориентацией и неравноправием ребер, инцидентным какому-либо атому, однако эти проблемы легко устраняются.

Молекула является полным инвариантом слоения. Поэтому с их помошью решается, например, такой вопрос: сколько существует различных лиувиллевых слоений на данной ориентируемой поверхности при условии, что число особых точек гамильтониана фиксировано? Чтобы это выяснить, достаточно перечислить все молекулы, которые могут быть скомбинированы из атомов, суммарная сложность которых равна числу особых точек гамильтониана. В качестве примера сформулируем следуюший результат.

ПРЕДЛОЖЕНИЕ 1. Число различных лиувиллевых слоений, имеющих не более 6 особых точек, на сфере и торе равно 8 и 14 соответственно.

Отметим, что если функция Морса $f$ является простой, т.е. на каждом особом уровне лежит ровно одна ее критическая точка, то имеется лишь два атома: 1) диск, расслоенный на концентрические окружности, и 2) окрестность восьмерки (рис. 1, (a) и (б)). В этом случае для описания слоения достаточно графа Риба, ребра которого снабжены ориентацией, показываюшей направление роста функции $f$. Хорошо известно, что с помошью малого возмущения произвольная функция Морса может быть сделана простой. Однако, иногда гамильтонова система обладает некоторой дополнительной дискретной симметрией, которая запрешает разведение особых точек на разные уровни. Поэтому сложные атомы действительно возникают в реальных задачах и их изучение тоже представляет интерес.

Отметим, кроме того, недавнее наблюдение, сделанное В.В. Шарко и А. А. Ошемковым и демонстрирующее связь сложных атомов с еще одной классической задачей, а именно, с классификациейпотоков Морса-Смейла на двумерных ориентируемых поверхностях. Выделим среди них так называемые градиентоподобные потоки, т.е. потоки Морса-Смейла без замкнутых траекторий.

Как и в гамильтоновом случае, они могут быть заданы с помошью некоторой функшии Морса $f$ на двумерной поверхности $M^{2}$, снабженной римановой метрикой, и имеют вид $v=\operatorname{grad} f$. Однако здесь в отличие от гамильтонова случая связь функции $f$ со структурой траекторий не является непосредственной: одна и та же функция при изменении метрики может давать траекторно неэквивалентные градиентные потоки, а один и тот же поток может отвечать топологически различным функциям.

Классификация градиентоподобных потоков на двумерных поверхностях с точностью до топологической траекторной эквивалентности хорошо известна (М. Пейксото [10]), однако их инварианты, предложенные в [10], довольно громоздки. Оказьвается, задача классификации таких потоков фактически равносильна классификации атомов.

ПРЕДЛОЖЕнИЕ 2. Существует естественное взаимнооднозначное соответствие между атомами и градиентоподобными потоками на двумерных ориен- 
тируемьх поверхностях, рассматриваемыми с точностью до топологической траекторной әквивалентности (без учета направлений на траекториях).

ЗАмечАниЕ. "Без учета направлений на траекториях" означает, что мы считаем эквивалентньми потоки, получаюшиеся друг из друга изменением ориентации на всех траекториях. Если придерживаться стандартного определения траекторной эквивалентности, то в этом утверждении вместо атомов следует рассматривать так называемые $f$-атомы (см. [9]).

Соответствие между атомами и градиентоподобньми потоками является следствием теоремы Мейера [11], которая утверждает, что для каждого градиентоподобного потока $v$ на двумерной поверхности найдется функция Морса $f_{v}$, имеющая ровно три критических значения (минимум, максимум и седловое значение), такая, что ее градиентный поток $\operatorname{grad} f_{v}$ топологически траекторно эквивалентен потоку $v$. При этом два потока $v_{1}$ и $v_{2}$ топологически траекторно эквивалентны тогда и только тогда, когда отвечаюшие им функции $f_{v_{1}}$ и $f_{v_{2}}$ эквивалентны топологически. Теперь остается заметить, что в том случае, когда все седловые точки функции Морса $f_{v}$ лежат на одном и том же особом слое, топология слоения поверхности $M^{2}$ на линии уровня функции $f_{v}$ однозначно определяется структурой этого особого слоя (т.е. соответствующим атомом).

Таким образом, зная список атомов фиксированной сложности, мы, в частности, автоматически получаем список градиентоподобных потоков на двумерных поверхностях с фиксированньм числом особых точек.

Как мы видим, в случае одной степени свободы описание лиувиллевых слоений гамильтоновых систем с морсовскими гамильтонианами не очень сложно и сводится к классификации так назьваемых атомов. Никаких топологических препятствий к полной интегрируемости здесь нет.

\section{Особенности коразмерности один}

Рассмотрим теперь случай симплектического многообразия $\left(M^{2 n}, \omega\right)$ произвольной размерности с интегрируемой гамильтоновой системой, отвечающей гамильтониану $H$ и обладающей независимыми интегралами $f_{1}, f_{2}, \ldots, f_{n}$. Рассмотрим соответствующее отображение момента $\mathscr{F}=\left(f_{1}, \ldots, f_{n}\right): M^{2 n} \rightarrow \mathbb{R}^{n}$ и множество его особых точек $K=\left\{x \in M^{2 n} \mid \operatorname{rank} d \mathscr{F}(x)<n\right\}$. Это множество допускает естественную стратификацию $K=K_{n-1} \cup K_{n-2} \cup \cdots \cup K_{0}$, где $K_{i}=\left\{x \in M^{2 n} \mid \operatorname{rank} d \mathscr{F}(x)=i\right\}$. Мы будем говорить, что особые точки из множества $K_{i}$ имеют коранг $i$.

Рассмотрим особьй слой $L$. Мы будем говорить, что отвечающая ему особенность лиувиллева слоения имеет коразмерность $k$, если $k$ - максимальньй коранг особых точек, содержащихся в $L$.

В этом разделе мы рассмотрим особенности коразмерности один. Среди таких особенностей выделяется класс невырожденных. Формальное их определение таково. Пусть $x \in K_{n-1}$ - особая точка коранга один. Рассмотрим подпространство $L \subset T_{x} M^{2 n}$, порожденное косыми градиентами функций $f_{1}, \ldots, f_{n}$, и его косоортогональное дополнение $\widetilde{L}=\left\{\xi \in T_{x} M^{2 n} \mid \omega(\xi, \underset{\sim}{)}=0 \forall \eta \in L\}\right.$. Поскольку функции $f_{1}, \ldots, f_{n}$ коммутируют, то $L$ изотропно и $L \subset \widetilde{L}$, причем $\operatorname{dim} \widetilde{L}-\operatorname{dim} L=2$. Дифференциалы $d f_{1}, \ldots, d f_{n}$ линейно зависимы в точке $x$, поэтому существует нетривиальная линейная комбинация $\lambda_{1} d f_{1}(x)+\cdots+\lambda_{n} d f_{n}(x)$, равная нулю. Рассмотрим форму 
$h=\lambda_{1} d^{2} f_{1}(x)+\cdots+\lambda_{n} d^{2} f_{n}(x)$. Она корректно определена, и, как нетрудно видеть, подпространство $L$ лежит в ее ядре. Поэтому $h$ задает корректно определенную форму на двумерном фактор-пространстве $\widetilde{L} / L$. Точка $x$ называется невырожденной, если эта форма невырождена. Особый слой $L$ называется невырожденным, если все особые точки, содержашиеся в нем, невырождены.

Аналогичным образом можно определить невырожденные особые точки произвольного коранга. Например, если $x \in K_{0}$ (т.е. коранг максимален), то дифференциалы всех функций $f_{1}, \ldots, f_{n}$ обрашаются в нуль в точке $x$, и невырожденность определяется так. Рассмотрим 2-формы $d^{2} f_{1}(x), \ldots, d^{2} f_{n}(x)$ как элементы пространства симметричных форм $S^{2}\left(T_{x}^{*} M\right)$. Это пространство имеет естественную структуру симплектической алгебры Ли, и формы $d^{2} f_{1}(x), \ldots, d^{2} f_{n}(x)$ образуют в ней коммутативную подалгебру (поскольку функции $f_{1}, \ldots, f_{n}$ коммутируют между собой). Особая точка $x$ называется невырожденной, если эта подалгебра является подалгеброй Картана. В случае произвольного коранга сначала нужно провести редукцию, чтобы получить точку максимального коранга, а затем использовать данное вьше определение.

Наложим еще одно ограничение на класс рассматриваемых особенностей, которое ниже мы будем предполагать выполненньм, если не оговорено противное. Пусть $U(L)$ - некоторая регулярная насьщенная окрестность особого слоя $L$. Обозначим через $\Sigma_{L}$ множество $\mathscr{F}(U(L) \cap K)$, т.е. образ особых точек, попавших в $U(L)$. Пусть $x \in L$ - особая точка максимального коранга. Аналогичньм образом рассмотрим ее малую окрестность $V(x)$ и множество $\Sigma_{x}=\mathscr{F}(V(x) \cap K)$. Мы будем предполагать, что локально множества $\Sigma_{L}$ и $\Sigma_{x}$ совпадают (для произвольной точки $x \in L$ максимального коранга).

Как устроено слоение Лиувилля в окрестности невырожденной особенности коразмерности один? Отметим, что этот вопрос можно переформулировать несколько иначе в терминах перестроек торов Лиувилля. Рассмотрим бифуркационную диаграмму $\Sigma=\mathscr{F}(K) \subset \mathbb{R}^{n}$. Обычно она представляет собой стратифицированное многообразие, разбивающее пространство $\mathbb{R}^{n}$ на области регулярных значений. Чтобы попасть из одной области в другую, необходимо пересечь страт коразмерности один. В самом многообразии $M^{2 n}$, если мы будем следить за прообразом точки из $\mathbb{R}^{n}$, в момент пересечения страта произойдет бифуркация лиувиллевских торов. Задача состоит в том, чтобы описать типы таких бифуркаций (т.е. невырожденных бифуркаций коразмерности один). Эта задача была решена А. Т. Фоменко в [3], [6].

Было показано, что локальная структура особенности в этом случае может быть двух типов. Первый тип - это прямое произведение. Чтобы его описать, рассмотрим произвольный атом $P^{2}$ и тривиальное слоение Лиувилля $\mathscr{L}_{n-1}$ размерности $n-1$, т.е. прямое произведение $(n-1)$-мерного тора на $(n-1)$-мерньй диск. Тогда на прямом произведении $X^{2 n}=P^{2} \times \mathscr{L}_{n-1}$ возникает естественная структура лиувиллева слоения, слоями которого являются прямые произведения слоев из сомножителей.

Второй тип (называемый почти прямым произведением) тоже непосредственно связан с атомами и может быть смоделирован следуюшим образом. Предположим, что атом $P^{2}$ допускает симплектическую инволюцию $\tau: P^{2} \rightarrow P^{2}$, сохраняющую структуру лиувиллева слоения. Тогда эта инволюция может быть распространена на пря- 
мое произведение $M^{2 n}=P^{2} \times T^{n-1} \times D^{n-1}$ по следующей формуле:

$$
\widetilde{\tau}\left(x, \varphi_{1}, \ldots, \varphi_{n-1}, s_{1}, \ldots, s_{n-1}\right)=\left(\tau(x), \varphi_{1}+\pi, \varphi_{2}, \ldots, \varphi_{n-1}, s_{1}, \ldots, s_{n-1}\right),
$$

где $x \in P^{2},\left(\varphi_{1}, \ldots, \varphi_{n-1}\right) \in T^{n-1},\left(s_{1}, \ldots, s_{n-1}\right) \in D^{n-1}$. Легко видеть, что $\widetilde{\tau}$ является инволюцией без неподвижных точек, сохраняющей симплектическую структуру и структуру лиувиллева слоения на $X^{2 n}$, поэтому фактор-пространство $\widetilde{M}^{2 n}=M^{2 n} / \widetilde{\tau}$ также приобретает естественную структуру лиувиллева слоения (типа почти прямого произведения).

В обоих случаях мы получаем довольно просто устроенную модельную особенность лиувиллева слоения. Утверждается, что любая невырожденная особенность коразмерности один топологически эквивалентна одной из модельных.

Таким образом, мы видим, что классификация невырожденных особенностей коразмерности один (или, что то же самое, перестроек торов Лиувилля общего положения) по-существу сводится к классификации атомов.

\section{Случай двух степеней свободы, изоэнергетические поверхности, молекулы}

Рассмотрим теперь случай интегрируемой гамильтоновой системы с двумя степенями свободы. В этом случае для того чтобы система была интегрируемой, достаточно сушествования одного дополнительного интеграла $f$, независимого с интегралом энергии $H$. Пусть $Q^{3}=\{H=$ const $\}$ - неособая компактная изоэнергетическая поверхность интегрируемой системы $X_{H}$ и $\mathscr{L}$ - лиувиллево слоение на ней. Задача классификации таких слоений в предположении о невырожденности особенностей была решена в [7], [8]. Условие невырожденности в этом случае можно переформулировать следуюшим естественным образом. Рассмотрим ограничение интеграла $f$ на изоэнергетическую поверхность $Q^{3}$. Требуется, чтобы критические точки функции $\left.f\right|_{Q^{3}}$ образовывали набор невырожденных критических окружностей эллиптического или гиперболического типа.

Полным инвариантом лиувиллева слоения в этом случае является так называемая меченая молекула гамильтоновой системы (инвариант Фоменко-Цишанга), которая является аналогом описанной выше молекулы для случая одной степени свободы. Она представляет собой граф, являющийся базой слоения (т.е. граф Риба), каждой вершине которого сопоставлен некоторый 3-атом, задающий топологический тип соответствуюшего особого слоя. Поясним, что при сделанных предположениях все особенности рассматриваемого лиувиллева слоения имеют коразмерность один и невырожденны, поэтому их классификация нам уже известна. Кроме этой информации инвариант $W^{*}$ включает набор числовых меток, показьвающий правила склейки трехмерного многообразия $Q^{3}$ из отдельных 3 -атомов.

В работе [8] был доказан следующий результат: две интегрируемые системы лиувиллево эквивалентны на своих изоэнергетических поверхностях тогда и только тогда, когда их меченые молекулы совпадают. Кроме того, было показано, что любая абстрактно заданная молекула может быть реализована как инвариант подходящей интегрируемой гамильтоновой системы.

После того как топология лиувиллевых слоений на трехмерных поверхностях была описана, оказалось возможньм выделить из класса $(M)$ всех замкнутых трехмерных 
многообразий те многообразия, которые могут служить изоэнергетическими поверхностями интегрируемых гамильтоновых систем, и получить тем самым новые топологические препятствия к интегрируемости. Обозначим подкласс таких многообразий через $(H)$. Более строго, мы полагаем, что $Q^{3} \in(H)$, если существует интегрируемая гамильтонова система, для которой $Q^{3}$ является неособой изоэнергетической поверхностью, причем ограничение дополнительного интеграла $f$ на $Q^{3}$ удовлетворяет условию невырожденности.

ЗАмечание. Как было показано С. В. Матвеевым и А. Т. Фоменко, условие невырожденности здесь можно сушественно ослабить, потребовав лишь, чтобы функция $\left.f\right|_{Q^{3}}$ была ручной.

Теорема 1 [3], [6]. Трехмерное многообразие $Q$ принадлежсит классу $(H)$ тогда и только тогда, когда $Q$ является граф-многообразием.

Известно, что класс $(W)$ графф-многообразий, введенных и изученных Вальдхаузеном в [12], не совпадает (сушественно меньше) с классом $(M)$. Например, ни одно гиперболическое 3 -многообразие не лежит в $(W)=(Q)$, в частности, гиперболичность является препятствием к интегрируемости. С другой стороны, как было показано С.В. Матвеевьм и А.Т. Фоменко, любое многообразие $Q \in(M)$ может быть изоэнергетической поверхностью некоторой (необязательно интегрируемой) гамильтоновой системы с двумя степенями свободы.

Можно ли сказать что-либо о топологических препятствиях к интегрируемости на всем четырехмерном симплектическом многообразии, используя описанные выше инварианты лиувиллевых слоений? Пока этот вопрос остается открытым, но имеются некоторые соображения и результаты, которые могут оказаться полезньми при его исследовании. Рассмотрим гамильтониан $H$ на всем четырехмерном симплектическом многообразии и все его изоэнергетические поверхности $Q_{h}^{3}=\{H(x)=h\}$. Будем менять уровень энергии $h$ и следить за изменением топологии лиувиллева слоения на $Q_{h}^{3}$. При некоторых значениях параметра $h$ будет происходить бифуркация слоения, другими словами, скачкообразное изменение молекулы системы, которое условно можно назьвать “химической реакцией” или хирургией. Можно описать в некотором смысле типичные бифуркации-реакции (см. [13]-[15]) и с их помощью попытаться представить себе, какие четырехмерные симплектические многообразия могут появиться в результате их последовательного применения. Справедлив, в частности, следуюший результат.

Теорема 2 (Нгуен Тьен Зунг, [15]). Фундаментальная группа симплектического многообразия не является препятствием $\kappa$ интегрируемости. Другими словами, для любой конечно-порожденной группь $G$ можно построить четьрехмерное компактное симплектическое многообразие $\left(M^{4}, \omega\right)$ с интегрируемой гамильтоновой системой $X_{H}$ такое, что $\pi_{1}\left(M^{4}\right)=G$.

ЗАМЕЧАНИЕ. В этом Утверждении имеется в виду, что особенности соответствующего лиувиллева слоения на $M^{4}$ будут иметь достаточно простую структуру. Они исчерпываются некоторым явно указьваемым конечньм списком. 


\section{Круговые молекулы и гипотеза Фоменко}

Возникает естественный вопрос: можно ли использовать те же самые инварианты (атомь и молекуль) для описания и классификации более сложных особенностей (например, вырожденных или особенностей произвольной коразмерности)? Оказьвается, что это действительно возможно.

Чтобы объяснить основную идею, рассмотрим случай двух степеней свободы. Пусть, как и вьше, $H$ - интеграл гамильтоновой системы, $f$ - ее дополнительный интеграл и $\mathscr{F}=(H, f): M^{4} \rightarrow \mathbb{R}^{2}$ - соответствующее отображение момента. Рассмотрим множество особых точек отображения момента $\mathscr{F}$

$$
K=\left\{x \in M^{4} \mid \operatorname{rank} d F(x)<2\right\}
$$

и бифуркационную диаграмму $\Sigma=\mathscr{F}(K) \subset \mathbb{R}^{2}$. Обычно бифуркационная диаграмма $\Sigma$ представляет собой объединение гладких кривых (которые соответствуют невырожденным особенностям коразмерности один) и некоторых особых точек бифуркационной диаграммы (которые соответствуют более сложным особенностям).

ОПРЕДЕЛЕНИЕ 4. Особая точка $y \in \Sigma \subset \mathbb{R}^{2}$ назьвается изолированной, если для любого достаточно малого $\varepsilon>0$ окружность $\gamma_{\varepsilon}$ с центром в точке $y$ и радиусом $\varepsilon$ пересекает бифуркационную диаграмму $\Sigma$ трансверсально и только в неособых точках.

Рассмотрим прообраз $Q_{\gamma_{\varepsilon}}^{3}=\mathscr{F}^{-1}\left(\gamma_{\varepsilon}\right)$. Легко видеть, что он представляет собой трехмерное гладкое многообразие, расслоенное на торы Лиувилля, причем все особенности этого слоения - невырождены. Мы можем интерпретировать $Q_{\gamma_{\varepsilon}}^{3}$ как изоэнергетическую поверхность и рассмотреть соответствуюшую меченую молекулу $W^{*}(y)$, которая задает структуру слоения Лиувилля на $Q_{\gamma_{\varepsilon}}^{3}$. Легко видеть, что $W^{*}(y)$ не зависит от выбора $\varepsilon$.

ОПРЕДЕЛЕНИЕ $5 . W^{*}(y)$ назьвается круговой молекулой (ассоциированной с особенностью слоения $\mathscr{L}$, соответствующей изолированной особой точке $y \in \Sigma$ ).

ГИПОТЕЗА ФОМЕНКО. Круговая молекула особенности является ее полным топологическим инвариантом.

Первоначально эта гипотеза была сформулирована для невырожденных особенностей. Однако она оказалась справедливой и в других ситуашиях, поэтому интересным был бы ответ на вопрос, для каких типов особенностей гипотеза Фоменко справедлива.

Эта конструкция и гипотеза могут быть обобщены на случай многих степеней свободы. Вместо молекулы нужно будет рассмотреть так называемую меченую сеть [16], которая показьвает типы и взаимное расположение особенностей коразмерности один в окрестности изучаемой особенности произвольной коразмерности.

Поясним, как гипотеза Фоменко может быть использована при изучении конкретных примеров интегрируемых гамильтоновых систем. В приложениях довольно трудно бывает описьвать особенности лиувиллевых слоений коразмерности больше единицы. С другой стороны, особенности коразмерности один находятся и описьваются намного проще. Если нам действительно удается это сделать, то гипотеза Фоменко говорит, что мы можем после этого однозначно восстановить локальную структуру всех остальных особенностей. 
Первые общие результаты, описываюшие структуру особенностей коразмерности два в случае двух степеней свободы, были получены Л. М. Лерманом и Я. Л. Уманским [17]. Затем в работах [18]-[20] этот класс особенностей был исследован с точки зрения инвариантов Фоменко. В результате былаполучена топологическая классификация невырожденных особенностей типа центр-центр, центр-седло, фокус и полный список особенностей типа седло-седло сложности один и два (сложностью назьвается число особых точек максимального коранга на особом слое). Были вычислены также соответствуюшие этим особенностям круговые молекулы [18], [20]. Оказалось, что гипотеза А. Т. Фоменко справедлива для всех этих случаев.

Теорема 3. В случае двух степеней свободы гипотеза Фоменко справедлива для невырожсденных особенностей типа центр-центр, иентр-седло, фокус, а такэсе для особенностей типа седло-седло сложсности один и два.

Более того, в случае двух степеней свободы эта гипотеза оказалась справедливой не только для невырожденных, но и для устойчивых особенностей коранга один. Список таких особенностей был недавно получен В. В. Калашниковым (мл.) [21]. Устойчивость в данном случае означает следуюшее.

Пусть $\mathscr{L}$ - слоение Лиувилля, отвечающее паре коммутирующих функций $H$ и $f$. Пусть $H_{\varepsilon}, f_{\varepsilon}$ - гладкое семейство коммутируюших функций (другими словами, гладкое возмушение исходной системы), $H=H_{0}, f=f_{0}$. Для каждого $\varepsilon$ мы имеем, следовательно, интегрируемую гамильтонову систему с гамильтонианом $H_{\varepsilon}$ и дополнительным интегралом $f_{\varepsilon}$ и можем рассмотреть соответствующее лиувиллево слоение $\mathscr{L}_{\varepsilon}$. Особенность слоения $\mathscr{L}=\mathscr{L}_{0}$ называется устойчивой (точнее, гомотопически устойчивой), если она не меняет свой топологический тип при описанных возмущениях, т.е. для достаточно малых $\varepsilon$ слоения $\mathscr{L}_{\varepsilon}$ и $\mathscr{L}$ изоморфны (полулокально, т.е. вблизи особого слоя).

Для устойчивых особенностей легко вычисляются круговые молекулы, в результате получается следующее утверждение.

Теорема 4. В случае двух степеней свободы гипотеза Фоменко справедлива для устойчивых особенностей ранга один.

\section{Невырожденные особенности в случае многих степеней свободы. Теорема о топологическом разложении}

Рассмотрим, наконец, случай $n$ степеней свободы и многомерные особенности лиувиллевых слоений. Попытаемся сначала смоделировать их каким-нибудь простым способом из элементарных особенностей, которые мы уже знаем (т.е. попросту из атомов). Простейший способ состоит в том, чтобы рассмотреть прямое произведение атомов $P_{1}, \ldots, P_{n}$. Действительно, если каждый атом рассматривать как тройку $\left(P_{i}, \omega_{i}, f_{i}\right)$, где $\omega_{i}$ - некоторая симплектическая структура на $P_{i}$, а $f_{i}: P_{i} \rightarrow \mathbb{R}-$ функция Морса, имеюшая ровно одну особую линию уровня, то на прямом произведении $X^{2 n}=P_{1} \times \cdots \times P_{n}$ можно рассмотреть симплектическую структуру $\Omega=$ $\omega_{1}+\cdots+\omega_{n}$. Функции $f_{1}, \ldots, f_{n}$ будут тогда коммутировать и задавать некоторое слоение Лиувилля на $X^{2 n}$. Произведение особых слоев $L_{i} \subset P_{i}$ будет особьм слоем этого слоения ранга ноль. Полученная особенность будет в естественном смысле иметь тип прямого произведения. 
Эту простую конструкцию можно обобшить, предположив, что на прямом произведении $X^{2 n}$ задано действие $\phi$ конечной группы $G$, удовлетворяюшее следуюшим свойствам:

1) действие свободно,

2) действие является симплектическим,

3 ) действие является покомпонентным, т.е. $\phi(g)\left(x_{1}, \ldots, x_{n}\right)=\left(\phi_{1}(g) x_{1}, \ldots\right.$, $\left.\phi_{n}(g) x_{n}\right)$, причем каждое действие $\phi_{i}$ на компоненте $P_{i}$ сохраняет функцию $f_{i}$ (и, следовательно, структуру слоения Лиувилля).

Если все эти условия вьполнены, то фактор-пространство $\widetilde{M}=M / G$ является симплектическим многообразием с естественной структурой лиувиллева слоения $\widetilde{\mathscr{L}}$. Тип полученной особенности мы будем называть почти прямьм произведением (прямое произведение естественно считать частным случаем).

Оказьвается (см. теорему 5 ниже), все невырожденные особенности лиувиллевых слоений топологически эквивалентны описанным вьше. Другими словами, любая особенность может быть получена из простейших атомов с помошью взятия прямого произведения и, быть может, последующей факторизации по действию конечной групшы. Прежде чем сформулировать этот результат в точной форме, сделаем еще два замечания о структуре атомов, участвуюших в конструкции. Во-первых, среди атомов $P_{1}$ могут быть тривиальные атомы без особенностей, т.е. прямые произведения $S^{1} \times D^{1}$. Кроме того, могут встречаться некоторые новые “четырехмерные атомы”, отвечаюшие особенностям типа фокус. Список таких “фокусных” атомов также хорошо известен [19], [23].

Теорема 5 (Нгуен Тьен Зунг, [22]). Любая невырожденная особенность лиувиллева слоения изоморфна особенности типа почти прямого произведения.

ЗАмЕчАниЕ. В этом утверждении изоморфизм понимается в смысле определения 2. Другими словами, топологическое разложение в произведение атомов не обязано быть симплектическим.

Таким образом, эта теорема, которая может рассматриваться как сингулярный аналог теоремы Лиувилля, показьвает, что структура невырожденных особенностей может быть полностью описана в очень простых терминах, а именно, в терминах атомов. В качестве примера рассмотрим структуру невырожденной особенности в случае двух степеней свободы при условии, что особьй слой содержит ровно одну критическую точку типа седло-седло. Согласно теореме о топологическом разложении окрестность этого особого слоя может быть представлена в виде произведения двух седловых атомов с последующей факторизацией. Оказьвается, в этом случае имеется ровно четыре возможности (необходимые для описания атомы изображены на рис. 1):

1) прямое произведение $B \times B$ двух атомов типа $B$;

2) $\left(B \times C_{2}\right) / \mathbb{Z}_{2}$, где группа $\mathbb{Z}_{2}$ действует на каждом из сомножителей как центральная симметрия;

3) $\left(B \times D_{1}\right) / \mathbb{Z}_{2}$, где группа $\mathbb{Z}_{2}$ действует на каждом из сомножителей как центральная симметрия;

4) $\left(C_{2} \times C_{2}\right) /\left(\mathbb{Z}_{2} \times \mathbb{Z}_{2}\right)$, где две образуюшие $\alpha$ и $\beta$ групшы $\mathbb{Z}_{2} \times \mathbb{Z}_{2}$ действуют следуюшим образом (см. рис. 2 , на котором дано несколько иное представление для атома $\left.C_{2}\right): \alpha$ действует как симметрия относительно оси $O x$ на первом 
экземпляре атома $C_{2}$ и как симметрия относительно оси $O z$ на втором, а вторая образуюшая $\beta$, наоборот, действует как симметрия относительно $O z$ на первом сомножителе и как симметрия относительно оси $O x$ на втором.

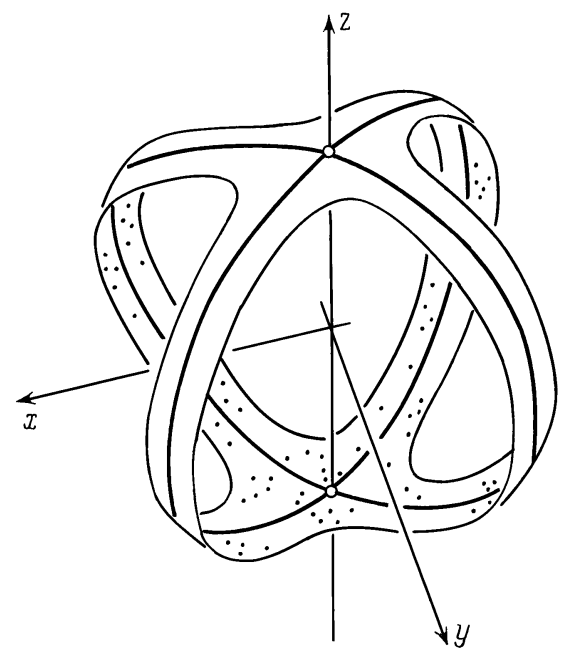

Рис. 2

Соответствующие круговые молекулы без меток изображены на рис. 3 (стандартные обозначения для атомов см. в [8]).
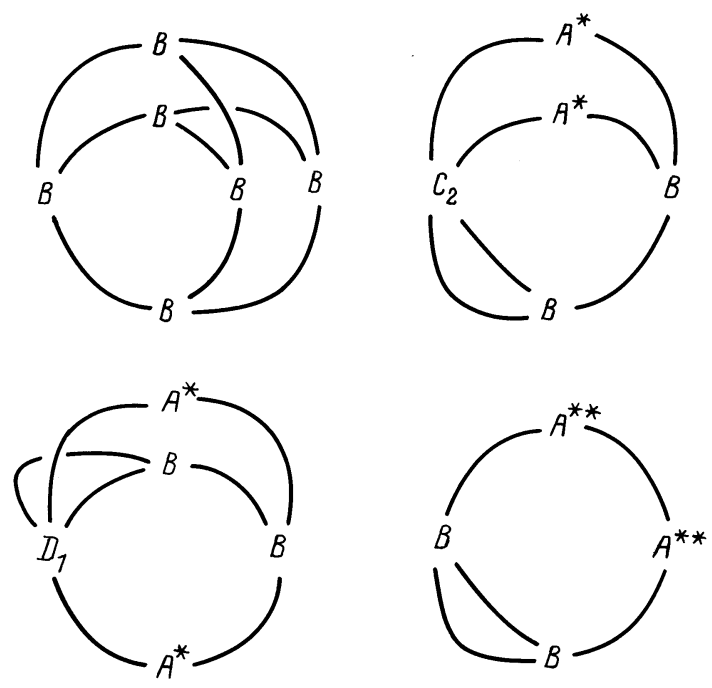

Рис. 3

Интересно, что первые три из четырех описанных особенностей встречаются в задачах классической механики (первая и третья - в интегрируемом случае Ковалев- 
ской, а вторая - в случае Горячева-Чапльгина [5], [18], [24]). Автору неизвестно, реализуется ли в интегрируемых системах механики и физики четвертая возможность. Во всяком случае ее нет в известных на сегодняшний день интегрируемых случаях динамики твердого тела, интегрируемых геодезических потоках на двумерных поверхностях и целой серии других систем, для которых инварианты Фоменко были вычислены в серии работ [24]-[26].

В [22] Нгуен Тьен Зунг также анонсировал следствие из теоремы о топологическом разложении, которое может быть переформулировано следуюшим образом: гипотеза Фоменко справедлива для всех невырожденных особенностей (доказательство этого факта пока не опубликовано).

\section{Траекторная классификация интегрируемых гамильтоновых систем}

Дальнейшее развитие подхода Фоменко привело к обнаружению новых траекторных инвариантов интегрируемых гамильтоновых систем с двумя степенями свободы и их классификации.

Напомним, что две динамические системы $\left(X_{1}, v_{1}\right)$ и $\left(X_{2}, v_{2}\right)$ называются топологически (гладко) траекторно эквивалентньми, если существует гомеоморфизм (дифофеоморфизм) $\xi: X_{1} \rightarrow X_{2}$, переводящий интегральные траектории первой системы в интегральные траектории второй (с сохранением их естественной ориентации, но, вообще говоря, без сохранения параметризации).

Ясно, что для нерезонансных систем отношение лиувиллевой эквивалентности слабее, чем отношение траекторной эквивалентности. Поэтому все описанные выше инварианты лиувиллевых слоений являются одновременно и траекторными инвариантами. Если рассматривать меченую молекулу интегрируемой системы как некоторьй ее портрет, то для построения полного траекторного инварианта нам нужно дополнить его новой информацией, сделав более детальньм. Решению этой задачи был посвящен цикл работ А. Т. Фоменко и автора [29]-[32], где была построена теория траекторной классификации интегрируемых гамильтоновых систем с двумя степенями свободы на изоэнергетических поверхностях. Было обнаружено, что природа траекторных инвариантов очень хорошо согласуется с представлением интегрируемой системы в виде молекулы, которое можно понимать как разбиение трехмерного изоэнергетического многообразия на атомы и ребра. Атомы - это малые окрестности особых слоев, а ребра - однопараметрические семейства торов Лиувилля без особенностей. Траекторные инварианты также разбиваются на два типа: реберные и атомные. Они описьвают соответственно поведение траекторий на ребрах и атомах молекулы. Кроме того, вся обшая схема построения полного траекторного инварианта системы (так называемой $t$-молекулы) оказалась весьма похожей на построение инварианта Фоменко-Цишанга.

Коротко полученные в работах [29]-[32] результаты можно сформулировать следующим образом. Пусть даны две интегрируемые по Лиувиллю гамильтоновы системы с двумя степенями свободы $v_{1}$ и $v_{2}$, ограниченные на свои неособые компактные изоэнергетические подмногообразия $Q_{1}$ и $Q_{2}$. Предполагается, что эти системы удовлетворяют некоторым естественньм ограничениям. Мы не будем здесь приводить полньй список таких ограничений, отсылая читателя к точным формулировкам в [29]-[32] и ограничившись замечанием, что подавляюшее большинство известных сегодня ин- 
тегрируемых гамильтоновых систем этим ограничениям удовлетворяет.

В [29]-[32] были описаны полные наборы инвариантов, позволяюшие сравнивать динамические системы $\left(v_{1}, Q_{1}\right)$ и $\left(v_{2}, Q_{2}\right)$ с точки зрения их траекторной эквивалентности, т.е. давать ответ на вопрос, сушествует ли гомеоморфизм (диффеоморфизм) $\xi: Q_{1} \rightarrow Q_{2}$, переводящий траектории первой системы в траектории второй с сохранением их естественной ориентации.

После построения общей теории классификашии возник естественньй вопрос: насколько эффективно траекторные инварианты интегрируемых гамильтоновых систем могут быть вычислены в конкретных задачах. Будет ли общая теория реально работать, если мы действительно захотим сравнить две конкретные системы и выяснить, эквивалентны ли они? В этой работе мы хотели бы на конкретном примере продемонстрировать, что ответ на этот вопрос является положительным (см. также [31]-[35]). Отметим, что сушествуют и другие методы, позволяюшие находить изоморфизмы между различньми интегрируемьми системами (см., например, [36]-[39]).

\section{Задача Якоби о геодезических на эллипсоиде и случай Эйлера в динамике твердого тела}

Мы рассмотрим здесь две знаменитые интегрируемые гамильтоновы системы: задачу Якоби о геодезических на эллипсоиде [40] и интегрируемьй случай Эйлера в динамике твердого дела [41], [42]. В работе [31] было показано сушествование траекторного гомеоморфизма между этими двумя системами. Здесь же, используя гладкие траекторные инварианты [29], мы покажем, что с гладкой точки зрения случай Эйлера и задача Якоби траекторно различны.

Рассмотрим эллипсоид $X$ в трехмерном евклидовом пространстве, задаваемьй уравнением

$$
\frac{x^{2}}{a}+\frac{y^{2}}{b}+\frac{z^{2}}{c}=1
$$

где $a<b<c$.

Геодезический поток на эллипсоиде - это гамильтонова система на кокасательном расслоении $T^{*} X$ со стандартной симплектической структурой, задаваемая гамильтонианом

$$
H(q, p)=\frac{1}{2} \sum g^{i j}(q) p_{i} p_{j}=\frac{1}{2}|p|^{2}
$$

где $g_{i j}(q)$ - индуцированная риманова метрика на $X,(q, p) \in T^{*} X, q \in X, p \in T_{q}^{*} X$. Изоэнергетическая поверхность $Q^{3}=\left\{2 H=|p|^{2}=1\right\}$ в этом случае является $S^{1}$-расслоением над $\mathrm{X}$ (расслоением единичных ковекторов). Геодезический поток на эллипсоиде допускает дополнительньй первьй интеграл

$$
f_{J}=a b c\left(\frac{x^{2}}{a^{2}}+\frac{y^{2}}{b^{2}}+\frac{z^{2}}{c^{2}}\right)\left(\frac{\dot{x}^{2}}{a}+\frac{\dot{y}^{2}}{b}+\frac{\dot{z}^{2}}{c}\right)
$$

Здесь $(\dot{x}, \dot{y}, \dot{z})$ - касательньй вектор к геодезической (мы отождествляем естественным способом касательные и кокасательные векторы). 
Вторая система (случай Эйлера) задается стандартными уравнениями ЭйлераПуассона и описывает движение твердого тела, закрепленного в центре масс

$$
\begin{gathered}
\frac{d K}{d t}=[K, \Omega], \\
\frac{d \gamma}{d t}=[\gamma, \Omega] .
\end{gathered}
$$

Здесь $K=\left(s_{1}, s_{2}, s_{3}\right)$ - вектор кинетического момента тела, $\Omega=\left(A s_{1}, B s_{2}, C s_{3}\right)-$ вектор его угловой скорости, $\gamma=\left(r_{1}, r_{2}, r_{3}\right)$ - единичньй вертикальный вектор (координаты этих векторов записаны в ортонормированном базисе, жестко связанном с телом, оси которого совпадают с главными осями инерции тела). Параметрами задачи служат обратные величины главных моментов инерции твердого тела $A, B, C$, $A<B<C$.

Хорошо известно, что эта система дифференциальных уравнений является гамильтоновой в шестимерном пространстве $\mathbb{R}^{6}\left(s_{1}, s_{2}, s_{3}, r_{1}, r_{2}, r_{3}\right)$, рассматриваемом как двойственное пространство алгебры Ли $e(3)=\operatorname{so}(3)+\mathbb{R}^{3}, s_{1} \in \operatorname{so}(3), r_{i} \in \mathbb{R}^{3}$. Напомним, что пуассонова структура задается при этом следующими формулами:

$$
\left\{s_{i}, s_{j}\right\}=\varepsilon_{i j k} s_{k}, \quad\left\{s_{i}, r_{j}\right\}=\varepsilon_{i j k} r_{k}, \quad\left\{r_{i}, r_{j}\right\}=0
$$

а гамильтониан системы имеет вид

$$
H=\frac{1}{2}\left(A s_{1}^{2}+B s_{2}^{2}+C s_{3}^{2}\right) .
$$

Напомним, что эта система уравнений Эйлера-Пуассона всегда имеет два дополнительных интеграла (функции Казимира пуассоновой структуры):

$$
\begin{aligned}
f_{0} & =|\gamma|^{2}, \\
g & =(K, \gamma) .
\end{aligned}
$$

Рассмотрим четырехмерное инвариантное подмногообразие $M^{4}=\left\{f_{0}=1, g=0\right\}$ и ограничим на него рассматриваемую систему. Скобка Пуассона индуцирует на $M^{4}$ некоторую симплектическую структуру $\omega$. Несложно показать, что получившееся симплектическое многообразие $\left(M^{4}, \omega\right)$ симплектоморфно кокасательному расслоению к двумерной сффере.

Таким образом, при сделанных выше ограничениях случай Эйлера является гамильтоновой системой с двумя степенями свободы на $T^{*} S^{2}$. Эта система интегрируема по Лиувиллю при помоши дополнительного интеграла

$$
f_{E}=s_{1}^{2}+s_{2}^{2}+s_{3}^{2}
$$

Изоэнергетическая поверхность $Q^{3}=\{2 H=1\}$ в случае Эйлера топологически устроена точно так же, как и в задаче Якоби, - это расслоение единичных (ко)векторов над сферой. 
В итоге обе системы (задачу Якоби и случай Эйлера) мы можем рассматривать как гамильтоновы системы на кокасательном расслоении к сфере. Более того, случай Эйлера при сделанных вьше ограничениях можно рассматривать как геодезический поток некоторой специальной метрики на сфере. Сфера с этой метрикой обычно называется сферой Пуанкаре. Сфера Пуанкаре и эллипсоид не изометричны.

Через $v_{J}(a, b, c)$ и $v_{E}(A, B, C)$ мы обозначим ограничения рассматриваемых систем Якоби и Эйлера на их изоэнергетические поверхности $Q_{J}=\left\{H_{J}=1\right\}$ и $Q_{E}=\left\{H_{E}=1\right\}$ соответственно, где $H_{J}$ и $H_{E}$ - гамильтонианы задачи Якоби и случая Эйлера, указанные выше.

ЗАмЕЧАНИЕ. Отметим, что в силу однородности гамильтонианов от выбора уровня энергии траекторное строение систем не зависит. Другими словами, при изменении уровня энергии каждая из систем остается траекторно эквивалентной исходной.

Итак, мы имеем две динамические системы $v_{J}(a, b, c)$ и $v_{E}(A, B, C)$, определенные на диффеоморфных изоэнергетических трехмерных многообразиях. Мы хотим теперь выяснить вопрос, насколько рассматриваемые системы похожи, являются ли они траекторно эквивалентными. Если да, то топологически или гладко?

Разумеется, это исследование нужно начинать с изучения лиувиллевых слоений этих систем. Формальный подсчет меченых молекул приводит к следующему результату.

ТеОРема 6. При любых значениях параметров системы $v_{J}(a, b, c)$ (задача Якоби) и $v_{E}(A, B, C)$ (случай Эйлера) лиувиллево әквивалентны.

Второй результат является следствием анализа топологических траекторных инвариантов для этих систем (т.е. явного подсчета $t$-молекул).

ТЕОРема 7. Существует взаимнооднозначное (с точностью до пропорииональности) соответствие между тройками параметров $a<b<c$ u $A<B<C$ такое, что для соответствующих троек динамические системы $v_{J}(a, b, c)($ задача Якоби) и $v_{E}(A, B, C)$ (случай Эйлера) топологически траекторно әквивалентнь.

Наконец, оказалось возможньм исследовать вопрос и гладкой траекторной эквивалентности случая Эйлера и задачи Якоби. Необходимые для сравнения систем гладкие траекторные инварианты могут быть в этом случае заданы явными формулами. Поскольку эти формулы довольно громоздки, то Х. Дуллиным и автором был проведен численньй анализ, приведший к следуюшему результату.

ТЕОРема 8. Ни при каких значениях параметров динамические системы (задача Якоби) и $v_{E}(A, B, C)$ (случай Эйлера) не являются гладко траекторно әквивалентными.

\section{СПИСОК ЛИТЕРАТУРЫ}

[1] Фоменко А. Т. Теория Морса интегрируемых гамилтоновых систем // Докл. АН СССР. 1986. T. 287. № 5. C. 1071-1075.

[2] Фоменко А. Т. Топология поверхностей постоянной энергии интегрируемых гамильтоновых систем и препятствия к интегрируемости // Изв. АН СССР. Сер. матем. 1986. Т. 50. C. 1276-1307. 
[3] Фоменко А.Т. Симплектическая геометрия. Методы и приложения. М.: Изд-во МГУ, 1988.

[4] Smale S. Topology and mechanics // Invent. Math. 1970. V. 10. P. 305-331.

[5] Харламов М. П. Топологический анализ интегрируемых задач динамики твердого тела. Л.: Изд-во ЛГУ, 1988.

[6] Fomenko A. T. Topological classification of all integrable Hamiltonian differential equations of general type with two degrees of freedom // The Geometry of Hamiltonian Systems. Proc. of a Workshop (June 5-16, 1989, Berkeley, USA). Berlin: Springer-Verlag, 1991. P. 131-339.

[7] Фоменко А. Т., Цишанг Х. Топологический инвариант и критерий эквивалентности интегрируемых гамильтоновых систем с двумя степенями свободы // Изв. АН СССР. Сер. матем. 1990. Т. 54. № 3. С. 546-575.

[8] Болсинов А. В., Матвеев С. В., Фоменко А.Т. Топологическая классификация интегрируемых гамильтоновых систем с двумя степенями свободы. Список систем малой сложности // УМН. 1990. Т. 45. № 2. С. 49-77.

[9] Ошемков А. А. Функции Морса на двумерных поверхностях. Кодирование особенностей // Труды МИРАН. 1994. Т. 205. С. 131-140.

[10] Peixoto M. M. On the classification of flows on 2-manifolds // Dynamical systems. Proc. Symp. Univ. of Bahia. New York: Academic Press, 1973. P. 389-419.

[11] Meyer K. R. Energy functions for Morse-Smale systems // Amer. J. Math. 1968. V. 90. № 4. P. 1031-1040.

[12] Waldhausen F. Eine Klasse von 3-Mannigfaltigkeiten // Invent. Math. 1967. V. 3. № 4 . P. 308-333; V. 4. № 2. P. 88-117.

[13] Болсинов А. В., Фоменко А. Т., Чанг К. Три типа бордизмов интегрируемых систем с двумя степенями свободы. Вычисление групп бордизмов // Труды МИРАН. 1994. Т. 205. C. $32-72$.

[14] Fomenko A.T. Theory of rough classification of integrable nondegenerate Hamiltonian differential equations on four-dimensional manifolds. Application to classical mechanics // Adv. Soviet Math. 1991. V. 6. P. 305-345.

[15] Nguen Tien Zung. Symplectic topology of integrable Hamiltonian systems // Thesis. Strasbourg, 1994.

[16] Fomenko A. T. The theory of invariants of multidimensional integrable Hamiltonian systems (with arbitrary many degrees of freedom). Molecular table of all integrable systems with two degrees of freedom // Adv. Soviet Math. 1991. V. 6. P. 1-36.

[17] Lerman L. M., Umanskii Ya. L. Structure of a Poisson action of $\mathbb{R}^{2}$ on a four-dimensional symplectic manifold. I, II // Selecta Math. Soviet. 1987. V. 6. № 4. P. 365-396; 1988. V. 7. № 1. P. 39-48.

[18] Bolsinov A. V. Methods of calculation of the Fomenko-Zieschang invariant // Adv. Soviet Math. 1991. V. 6. P. 147-183.

[19] Матвеев В.С. Интегрируемые гамилтоновы системы с двумя степенями свободы. Топологическое строение насыщенных окрестностей точек типа фокус-фокус и седло-седло // Матем. сб. 1996. Т. 187. № 4. С. 29-58.

[20] Матвеев В.С. Вычисление значений инварианта Фоменко для точки типа седло-седло интегрируемой гамильтоновой системы // Труды семинара по вект. и тенз. анализу. М.: Изд-во МГУ, 1993, Вып. 24. С. 75-104.

[21] Kalashnikov V. V. A class of generic integrable Hamiltonian systems with two degrees of freedom // Preprint 907. Univ. of Utrecht, Dept. of Math, 1995.

[22] Nguen Tien Zung. Symplectic topology of integrable Hamiltonian systems, I: ArnoldLiouville with singularities // Compositio Math. (to appear).

[23] Nguen Tien Zung. A note on focus-focus singularities // Differential Geom. Appl. (to appear).

[24] Oshemkov A. A. Fomenko invariants for the main integrable cases of the rigid body motion equations // Adv. Soviet Math. 1991. V. 6. P. 67-146. 
[25] Селиванова Е.Н. Классификация геодезических потоков лиувиллевых метрик на двумерном торе с точностью до топологической эквивалентности // Матем. сб. 1992. Т. 183. № 4. C. $69-86$.

[26] Nguen Tien Zung, Polyakova L.S. A topological classification of integrable geodesic flows on the two-dimensional sphere with an additional integral quadratic in the momenta // J. Nonlinear Sci. 1993. V. 3. № 1. P. 85-108.

[27] Duistermaat J. J. On global action-angle variables // Comm. Pure Appl. Math. 1980. V. 33. P. $678-706$.

[28] Audin M. The Topology of Torus Action on Symplectic Manifold. Basel: Birkhäuser, 1991.

[29] Болсинов А.В. Гладкая траекторная классификация интегрируемых гамильтоновых систем с двумя степенями свободы // Матем. сб. 1995. Т. 186. №1. С. 3-28.

[30] Болсинов А. В., Фоменко А. Т. Траекторная эквивалентность интегрируемых гамильтоновых систем с двумя степенями свободы. Теорема классификации I, II // Матем. сб. 1994. Т. 185. № 4. С. 27-80; 1994. Т. 185. № 5. С. 27-28.

[31] Болсинов А. В., Фоменко А. Т. Траекторная классификация геодезических потоков двумерных эллипсоидов. Задача Якоби траекторно эквивалентна интегрируемому случаю Эйлера в динамике твердого тела // Функц. анализ и его прил. 1995. Т. 29. № 3. С. 1-15.

[32] Болсинов А. В., Фоменко А. Т. Траекторные инварианты интегрируемых гамильтоновых систем. Случай простых систем. Траекторная классификация систем типа Эйлера в динамике твердого тела // Изв. РАН. Сер. матем. 1995. Т. 59. № 1. С. 65-102.

[33] Орел О. Е. Вектор вращения для интегрируемшхх задач, сводящихся к уравнениям Абеля. Траекторная классификация систем Горячева-Чаплыгина // Матем. сб. 1995. Т. 186. № 2. C. $105-120$.

[34] Орел О.Е., Такахаши Ш. Траекторная классификация интегрируемых задач Лагранжа и Горячева-Чапльпина методами компьютерного анализа // Матем. сб. 1996. Т. 187. № 1. С. $95-112$.

[35] Селиванова Е. Н. Траекторные изоморфизмы лиувиллевых систем на двумерном торе // Матем. сб. 1995. №10. С. 141-160.

[36] Adler M., van Moerbeke P. The Kowalewski and Henon-Heiles motions as Manakov geodesic flows on SO(4). A two-dimensional family of Lax pairs // Comm. Math. Phys. 1988. V. 113. № 4. P. 659-700.

[37] Veselov A.P. Two remarks about the connection of Jacobi and Neumann integrable systems // Math. Z. 1994. V. 216. P. 337-345.

[38] Knorrer H. Geodesics on quadrics and a mechanical problem of C. Neumann // J. Reine Angew. Math. 1982. V. 334. P. 69-78.

[39] Козлов В.В. Две интегрируемые задачи классической динамики // Вестник МГУ. Сер. 1. Матем., мех. 1981. № 4. С. 80-83.

[40] Якоби К. Лекции по динамике. М.-Л., 1936.

[41] Архангельский Ю. А. Аналитическая динамика твердого тела. М.: Наука, 1977.

[42] Козлов В. В. Методы качественного анализа в динамике твердого тела. М.: Изд-во МГУ, 1980 .

Московский государственньй

Поступила в редакцию

университет им. М.В. Ломоносова

19.06.1997

E-mail: bols@difgeo.math.msu.su 